recorded by Jomini. I have elsewhere ${ }^{2}$ analysed this case, which is probably one of gross congenital abnormality, with secondary adhesion processes. It cannot be allowed as a hernia of this class.

The only two authentic cases, so far as I am aware, are recorded-the tirst by Mr. F. S. Eve, and the second by Mr. McAdam Eccles. Mr. Eve's case was described and figured in the British MEdical Journal of June, 1885 , and I must refer the reader to this article for full particulars. In that case the most likely explanation of the history seems to me to be this. There had been originally a common mesentery for the jejunum, ileum, cæcum, and ascending colon. The splenic flexure and the descending colon became fixed in the usual manner by adhesion of the left or posterior layer of the original descending mesocolon to the parietal peritoneum of the posterior abdominal wall. As a result of peritoneal adhesion, most probably pathological, the cæcum and ascending colon had adhered to the descending colon, and the sigmoid and the united gut had been dragged over to the right, laying bare and rendering patent the orifice of the intersigmoid fossa.

Mr. McAdam Eccles's case occurred in 1895 . I am indebted to him for a reprint of his account in the St. Bartholomew's Hospital Reports. The symptoms were those of acute intestinal obstruction. On opening the abdomen "a tightly constructing edge, which afterwards proved to be the margin of the aperture of the intersigmoid fossa," was revealed. A loop of gut 4 inches in length was nipped in the fossa, and about half an inch of it was gangrenous. The loop was removed and the ends stitched by Maunsell's method. The patient died of exhaustion. At the post-mortem examination there were signs of "some general peritonitis, most marked in the region of the sigmoid flexure, which was itself thrown over to the right side of the body."

These two cases illustrate as well as possible two quite opposite conditions. In the one there was congenital abnormality, gross in extent, and peritoneal adhesion, probably pathological and extrauterine, and in the other a peritoneal cavity in all respects normal.

\section{Hernia into the Foramen OF Winslow.}

This form of hernia is very uncommon. I have been able to find only 8 cases recorded, 4 very imperfectly by Rokitansky, Treitz, Moir, and Novello, and 4 more fully by Blandin, Majoli, Elliot-Square, and Treves. After reading carefully the reports of these cases, it seems to me almost certain that in order to permit of the occurrence of this form of hernia, there must be some extreme congenital abnormality. In the carefully-recounted cases this has been observed. Thus in Treitz's case there was " a common mesentery/for the intestine from the duodenum to the rectum." In the cases recorded by Elliot-Square and Treves there was an absence of the secondary fusion process between the ascending colon and the posterior abdominal wall. The causes leading to the formation of this form of hernia are probably:

I. A common mesentery for the whole intestine. Rogie has collected 53 examples of this, and he has not included all the cases.

2. Absence of the secondary fusion of the ascending colon to the posterior abdominal wall.

3. Abnormally large size of the foramen of Winslow.

4. Abnormal length of mesentery, and, consequently, undue mobility of the intestine.

In the absence of one or other of these abnormalities, the occurrence of the hernia may be considered almost a physical impossibility.

\section{Symptoms.}

On analysing the series of cases, it seems that a hernia into the foramen of Winslow on becoming strangulated presents a fairly well-defined group of symptoms. These are :

1. Pain.-This is generally epigastric. Square speaks of 'pain over the ensiform cartilage and immediately below it "; Treves, of "pain like cramp atand above the umbilicus"; Majoli, of "a painful rounded tumour" in the epigastrium.

2. Tumour.-An epigastric tumour has usually been observed. Thus, Majoli says, " a persistent bulging of the anterior abdominal wall in the epigastric region "was present. Treves speaks of "a conspicuous bulging of the anterior abdominal wall in the epigastric and hypochondriac regions." Square says "the umbilicus was somewhat prominent."

3. Percussion.-A tumour when present is dull on light percussion, but deep percussion sounds a more resonant note.

It is interesting to note that there are no recorded symptoms of pressure on the hepatic artery, portal vein, or bile duct.

1 On Retroperitoneal Hernia. London: Baillière, Tindall, and Cox. 1899.

\section{THE THERAPEUTICS OF HEAT AND LIGHT AND} ELECTRIC HEAT AND LIGHT BATHS.

BY H. LEWIS JONES, M.D., F.R.C.P.,

Medical Officer in Charge of the Electrical Department, St. Bartholomew's Hospital.

WITH the house-to-house distribution of electric currents from central supply stations the application of the current from the mains to purposes other than the lighting of rooms will gradually become more general. Especially does the use of the current as a source of heat recommend itself on the score of convenience and ease of application, and a number of appliances for adapting electric heating to medical practice have lately been contrived. In some of these appliances incandescent lamps are used as the thermal generators, and certain special advantages have been claimed for them on the ground that in an incandescent lamp the heat emitted is from a source at a very high temperature, and is radiated rather than conducted, and that the light which is also given out by the lamps may have a beneficial effect. Thus Winternitz ${ }^{1}$ has a paper on this subject with the title of Electric Light Baths, and with figures of some of the apparatus he uses. These consist of wooden boxes or cabinets arranged for the reception of a patient's body or limbs and fitted within with incandescent electric lamps and reflectors. In them the patient can be subjected to the light (and the heat) given out by the lamps. The diseases in which the treatment is said to have been found useful are rheumatism, asthma, anæmia, neurasthenia, and obesity.

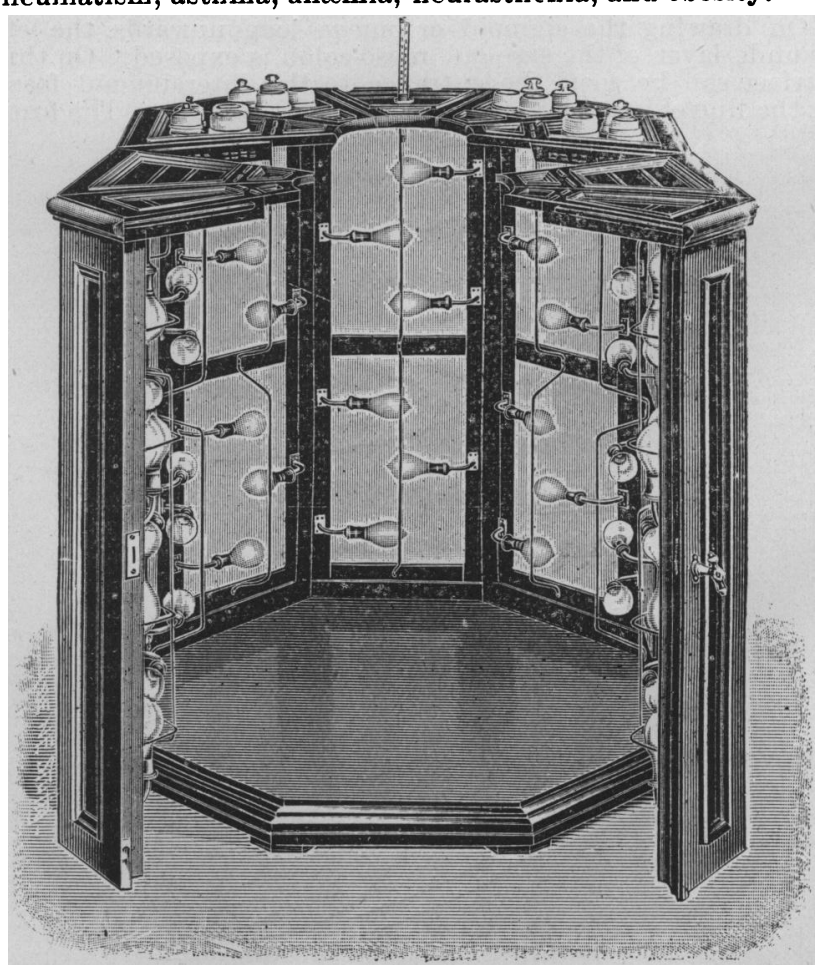

Fig. x.-Electric light bath of Winternitz. 
Winternitz states that the patients perspire profusely while in the cabinets, and the rapidity with which the perspiration sets in is specially insisted on by him. He says that after five minutes' exposure to the the air in the cabinets is not more than $81^{\circ} \mathrm{F}$

Kellogg, of Battle Creek, U.S.A., made experiments with the same kind of apparatus in 1894, and arrived at the same results. He seems to have recognised that the effects noticed were mainly due to radiant heat, and compares the action of radiant heat from a source at a high temperature with that of the heat of lower grade which is employed in the hot room of a Turkish bath. Thus, he says the degree of perspiration obtained with an air temperature of $85^{\circ} \mathrm{F}$. in the electric cabinet was equal to that produced in a Turkish bath at $106^{\circ} \mathrm{F}$.

This comparison must, however, be imperfect in the absence of data as to the degree of humidity of the atmosphere surrounding the patients in the two cases. The atmosphere inside a small closed box is entirely unlike that of the hot room of a Turkish bath, which is properly ventilated and supplied with volumes of hot dry air. In spite of this objection, one may agree with the views of Kellogg and of Winternitz that the use of incandescent lamps as described does present certain advantages, namely, that the radiant heat penetrates the tissues more deeply, that the effect is more stimulant, and that perspiration is more quickly induced, also that the comparative coolness of the air about the patient is an advantage. It is uncertain whether the light emitted by the lamps plays an important part or not. Under the name of the "Dowsing radiant heat bath" an apparatus of a similar kind has lately been introduced in this country by a limited company, and establishments for the treatment of patients have been set up in London and in several provincial towns. The conditions which seem specially suited for treatment by radiant heat are just those which are relieved by the application of heat in other ways, such as rheumatic and gouty affections of the joints or muscles, sprains, myalgias, and other painful states. It does not appear at present that radiant heat has any special therapeutic properties which cannot be had from the older methods of applying warmth.

There is another contrivance for the production of heat electrically which is sometimes useful medically, and that is the electrotherm, which was noticed in the BRITISH MEDICAL Journal for November gth, 1895 .

It consists of sheets of asbestos cloth interwoven with a fine wire, and enclosed in a cover forming a flexible pad about $\mathbf{r}_{4}$ inches square. When attached to a wall plug the current passes through the wire and warms it, the heat being regulated by a switch. For all conditions which can be relieved by the local application of heat it is admirable, and many such conditions relief of sciatica or lumbago I have found it useful. The electrotherm, with certain minor improvements, promises to be a useful domestic ap pliance as a foot warmer, or for any of the conditions now met by mean of poultices, hot bottles, and the like. Its predominant merit consists in the fact that the turning of a switch is all thatis needed to make it ready and, when made ready, it goes on at full emciency until switched o again. The defect of the electrotherm as at present made lies in the weakness of the fine wire with which it is fitted. This wire tends to which it oxidised and brittle from the alternate heatings and cooling which it undergoes, and in time it breaks. If platinum wre were subst frst cost, but would give a durability which it lacks at present.

Another electrically-warmed appliance is the "Greville electrothermic generator."

It is heated by electricity. The heat,is generated by electricity passing through wires, and not through lamps as in the radiant heat apparatus of Dowsing.

When their fragility is not an obstacle, incandescent lamps afford at once the cheapest and the most efficient of thermal generators. Messrs. Arnold and Sons are now working out with me the details of an electrically-heated operating table in which incandescent lamps supply the heat.

The body of the table has the form of an air chamber of metal, which may be divided into separate sections for convenience. Six or eight sixteen-candle power lamps enclosed in this chamber will rapidly raise an operating table to the required temperature, and half that number will then maintain the temperature at a proper level when the rest have been switched off. Wherever electric light circuits exist, th

Some work has also been done upon the therapeutic effect of the light from arc lamps. For obtaining effects genuinely due to light as distinguished from radiant heat, the arc lamp should present great advantages by reason of the great intensity of its light, and of its richness in the more refrangible rays. The workmen in electric welding establishments, and in other places where they are brought into close proximity with arc lights, are apt to suffer severely from effects which very closely resemble those of sunburn, as experienced by mountaineers after exposure to the glare from sunlit snow. From investigations which have been made by several observers, it appears certain that these effects are really due to light and not to heat. It does not seem unreasonable to believe that good results might follow a systematic course of treatment by the light of the electric arc; and especial advantages might be expected from it in anæmia, because anæmia so commonly occurs in people who are more or less deprived of light.

Dr. Margaret Cleaves ${ }^{2}$ has lately written upon the therapeutic action of arc lamps :

She describes a cabinet for the reception of the patient with two arc lamps arranged outside so as to shine into the cabinet through glas windows which may be open or closed. The patient is nude, wears dark spectacles, and reclines on a couch between the lamps. She has observed an increase in the hæmoglobin and the red blood corpuscles after two weeks' treatment. Her chief attention seems to have been given to cases with chronic pulmonary complaints, such as phthisis, asthma, and bronchitis, in which she obtained results which seemed to show an immediate beneficial action from the electric light. In a case of psoriasis and one of eczematous ulcer the effect was prompt and good. She also quotes from Koslovski,s $\mathrm{La}$ Touche, and Below. The former had his attention drawn to the subject from statements as to good effects noticed by workmen in electric welding works, and had obtained cu
cases, mostly neuralgia, sciatica, lumbago, and the like.

More extended observations are called for, and it is not unlikely that in time the arc lamp may take a place in therapeutics, particularly in the gloom iand darkness of big cities.

Several independent writers have noted that the arc lamp, either by its light or by the emission of ozone or other vapours, has a very decided disinfecting or deodorising action upon noxious smells. Thus an arc lamp set up in a dark basement near offensive closets and latrines served to dissipate all bad odour completely from the place in which it was hung.

REFERENCES.

1 Aertzliche Polytechnik, October, 1808,2 New York Medical Journal, January 28 th, February 4 th, I8g9. 3 Revue Internationale d'Electrothénapie April, May, 1896, and March, 1898 .

\section{THE JOINTS OF MAMMALS CONTRASTED WITH THOSE OF MAN.*}

By F. G. PARSONS, F.R.C.S.

Lecturer on Comparative Anatomy and Elementary Biology, and Demonstrator of Anatomy at St. Thomas's Hospital Medical School, London.

LECTURE I.

In this lecture the temporo-maxillary joint, the clavicular articulations and the shoulder-joint were dealt with. In the temporo-maxillary the importance of the external lateral ligament was drawn attention to, and it was shown that the interarticular fibro-cartilage was absent in certain of the lower mammals. The varieties of the joint in the different orders of the Mammalia were explained. In the sterno-clavicular joint the presence and meaning of the meniscus were discussed, as well as the various modifications by which the clavicle is attached to the coracoid and acromial processes. In the shoulder-joint reasons were brought forward for believing that the middle gleno-humeral ligament is more or less well represented throughout the Mammalia, while the superior and inferior are peculiar to man. The coraco-humeral ligament is peculiar to the Primates.

LEOTURE II.

The elbow, wrist, and hip-joints were dealt with in the second lecture. In the elbow the contrast between the arrangement of bones and ligaments in animals which use their fore limbs for support and those which use them for other purposes was drawn attention to. The utility of the orbicular and the oblique humero-radial ligament in various orders was described. In the wrist-joint the arrangements were seen to be very varied. Sometimes a septum dividing the joint into radial and ulnar compartments was found; sometimes a triangular fibro-cartilage was present, though more often it was absent or merged in the lower part of the interosseous membrane; its uses and significance were discussed in some detail. In the hip the various thickenings of the capsule were shown to be characteristic of the Primates. The ligamentum teres was followed throughout the mammalian class, and its various modifications studied with a * Abstract of three lectures delivered before the Royal College of
Surgeons of England. 